\title{
STUDY OF THE RELATION BETWEEN PLASMA LEVEL OF VON WILLEBRAND FACTOR AND DIABETIC RETINOPATHY IN TYPE 2 DIABETES
}

Eman Yousef Moursi, Noha Mohamed Gaber Amin, Heba Sadek Kassab, Abdiwahab Noor Abdirahman

Department of Internal Medicine, Faculty of Medicine, Alexandria University

\section{INTRODUCTION}

Diabetes mellitus is characterized by organ dysfunction arising from the effects of chronic hyperglycemia. The chronic complications of diabetes are classified as macro and microvascular depending on the underlying pathophysiology. The microvascular triad of retinopathy, nephropathy and neuropathy is unique to diabetes. Chronic inflammation plays an essential role in the progression of diabetic microvascular complications. Recently, new markers have been studied in the pathogenesis of diabetes and its complications such as Von Willebrand factor. Von Willebrand factor also seems to be a reason for diabetic microangiopathy and its severity.

\section{AIM OF THE WORK}

The study was aimed at demonstrating the relationship between the levels of serum Von Willebrand and factor (vWF) and diabetic retinopathy, as an early predictor for developing diabetic retinopathy and its severity.

\section{SUBJECTS AND METHODS}

A cross-sectional study was conducted on 60 patients with diabetes mellitus and recruited from the Vitreo- retinal and diabetes Outpatient Clinic at Alexandria Main University Hospital.

Group (A): 20 patients, T2DM with no diabetic retinopathy.

Group (B): 20 patients, T2DM with non-proliferative diabetic retinopathy.

Group (C): 20 patients, T2DM with proliferative diabetic retinopathy.

All the study participants were subjected to full general history taking, physical and ophthalmological examinations. All patients with active infections or previous hepatic, renal, malignancy, hematological and previous ophthalmological surgery were excluded from the study.
The eye examination included slit lamp for the anterior segment, indirect ophthalmoscope examination of the fundus and fluorescein angiography when the media were clear enough.

Venous blood sample of $5 \mathrm{ml}$ were drawn from each patient and collected in a sterile Wasserman tube. The sumpe were centifuged to sepate plasta serum and stored in $-80^{c}$. Von Willebrand factor measurement was performed by commercially available ELISA kit.

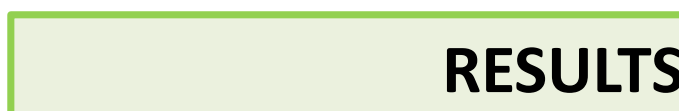

Table 1: Comparison between the three studied groups according to Von Willebrand factor level

\begin{tabular}{|c|c|c|c|c|c|c|c|c|c|c|}
\hline \multirow{3}{*}{$\begin{array}{l}\text { Von Willebrand } \\
\text { factor level }\end{array}$} & \multicolumn{10}{|c|}{$\begin{array}{l}\text { Eye examination } \\
\end{array}$} \\
\hline & \multicolumn{2}{|c|}{$\begin{array}{c}\text { Group A } \\
\text { No } \\
\text { retinopathy } \\
\quad(\mathbf{n}=\mathbf{2 0})\end{array}$} & \multicolumn{2}{|c|}{\begin{tabular}{|c|} 
Group B \\
$\quad$ Non \\
proliferative \\
retinopathy \\
$(\mathbf{n}=\mathbf{2 0})$ \\
\end{tabular}} & \multicolumn{2}{|c|}{$\begin{array}{c}\text { Group C } \\
\text { Proliferative } \\
\text { retinopathy } \\
(\mathbf{n}=\mathbf{2 0})\end{array}$} & \multicolumn{2}{|c|}{$\begin{array}{c}\text { Total sample } \\
(\mathbf{n}=6 \mathbf{6 0})\end{array}$} & \multicolumn{2}{|c|}{$\begin{array}{c}\text { Group B \& C } \\
(\mathbf{n}=40) \\
\text { (Diabetic } \\
\text { retinopathy) }\end{array}$} \\
\hline & No. & $\%$ & No. & $\%$ & No. & $\%$ & No. & $\%$ & No. & $\%$ \\
\hline $\begin{array}{l}\text { Normal } 47- \\
197 \%\end{array}$ & 16 & 80.0 & 4 & 20.0 & 3 & 15.0 & 23 & 38.3 & 7 & 17.5 \\
\hline Abnormal & 4 & 20.0 & 16 & 80.0 & 17 & 85.0 & 37 & 61.7 & 33 & 82.5 \\
\hline Min. - Max. & \multicolumn{2}{|c|}{$60.0-208.0$} & \multicolumn{2}{|c|}{$180.0-228.0$} & \multicolumn{2}{|c|}{$190.0-256.0$} & \multicolumn{2}{|c|}{$60.0-256.0$} & \multicolumn{2}{|c|}{$180.0-256.0$} \\
\hline Mean \pm SD. & \multicolumn{2}{|c|}{$119.05 \pm 51.34$} & \multirow{2}{*}{\multicolumn{2}{|c|}{$\frac{208.3 \pm 12.56}{2100}$}} & \multirow{2}{*}{\multicolumn{2}{|c|}{$\begin{array}{c}219.15 \pm 19.30 \\
218.5\end{array}$}} & \multirow{2}{*}{\multicolumn{2}{|c|}{$\begin{array}{c}182.17 \pm 55.36 \\
2020\end{array}$}} & & \\
\hline Median & & & & & & & & & \multicolumn{2}{|c|}{2140} \\
\hline Sig. bet. grps. & \multicolumn{6}{|c|}{$\mathrm{p}_{1}<0.001^{*}, \mathrm{p}_{2}<0.001^{*}, \mathrm{p}_{3}=0.176$} & & & & \\
\hline $\mathbf{p}_{\mathbf{n}}$ & & $01^{*}$ & 0.2 & & & & & & & \\
\hline \multicolumn{11}{|c|}{ 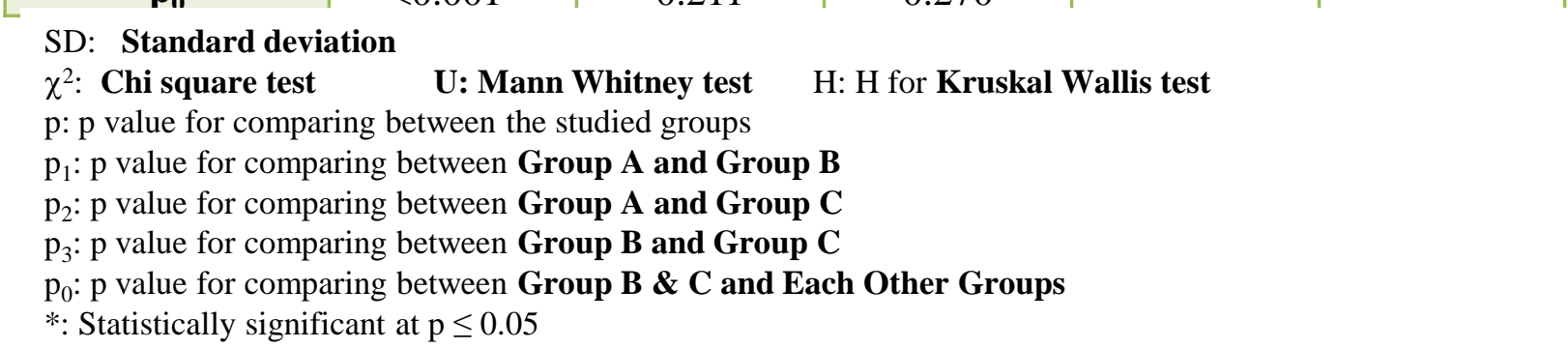 } \\
\hline
\end{tabular}

Table 2: Univariate and multivariate binary logistic regression for the parameters affecting diabetic retinopathy ( $\mathrm{n}=20$ vs. 40 )

\begin{tabular}{|c|c|c|c|c|}
\hline & \multicolumn{2}{|l|}{ Univariate } & \multicolumn{2}{|c|}{ "Multivariate } \\
\hline & OR (95\% C.I) & p & OR (95\% C.I) & $\mathbf{p}$ \\
\hline Age (years) & $1.312(0.987-1.744)$ & 0.061 & & \\
\hline Sex (females) & $0.740(0.252-2.175)$ & 0.584 & & \\
\hline BMI & $1.886(1.341-2.652)$ & $<0.001^{*}$ & $0.840(0.276-2.557)$ & 0.759 \\
\hline HbA1c & \begin{tabular}{c|}
$32.377(4.281-$ \\
$244.884)$
\end{tabular} & $0.001^{*}$ & $\begin{array}{l}\text { 313.9(3.088- } \\
31918.6)\end{array}$ & $0.015^{*}$ \\
\hline Duration & $\begin{array}{c}21.303(4.115- \\
110.286)\end{array}$ & $<0.001^{*}$ & $9.354(0.304-287.5)$ & 0.201 \\
\hline HTN & $\begin{array}{c}39.462(4.751- \\
327.753)\end{array}$ & $0.001^{*}$ & $\begin{array}{c}118.27(0.284- \\
49327.4)\end{array}$ & 0.121 \\
\hline UAC (Mg/g) & $1.046(1.012-1.082)$ & $0.008^{*}$ & $1.004(0.964-1.046)$ & 0.831 \\
\hline $\begin{array}{l}\text { Von Willebrand } \\
\text { Fartar }\end{array}$ & $\mid 1.078(1.014-1.145)$ & $0.016^{*}$ & $0.012(0.001-1.722)$ & 0.081 \\
\hline \multicolumn{5}{|c|}{$\begin{array}{l}\text { OR: Odd's Ratio } \quad \text { C.I: Confidence interval } \\
\text { \#: All variables with } \mathrm{p}<0.05 \text { was included in the multivariate }\end{array}$} \\
\hline \multicolumn{5}{|c|}{ CONCLUSION } \\
\hline
\end{tabular}

- This study showed that the impact of serum Von Willebrand factor on development of diabetic retinopathy in type 2 diabetes is more pronounced than previously thought.

- The high mean serum vWF level noticed in subjects with type 2 diabetes with diabetic retinopathy (than the subjects with no retinopathy) may be explained by the chronic inflammatory state in type $2 \mathrm{DM}$.

Despite Von Willebrand factor being higher in Type 2 DM subjects with diabetic retinopathy, multivariate analysis showed that $\mathrm{vWF}$ was not significant independent risk factor associated with diabetic retinopathy in type $2 \mathrm{DM}$.

20210Alexandria Faculty of Medicine
CC-BY-NC

